\title{
Einstein-Podolsky-Rosen paradox and quantum steering in pulsed optomechanics
}

\author{
Q. Y. $\mathrm{He}^{1,2}$ and M. D. Reid ${ }^{1}$ \\ ${ }^{1}$ Centre for Quantum Atom Optics, Swinburne University of Technology, Melbourne, Australia \\ ${ }^{2}$ State Key Laboratory of Mesoscopic Physics, School of Physics, Peking University, Beijing 100871, China
}

\begin{abstract}
We describe how to generate an Einstein-Podolsky-Rosen (EPR) paradox between a mesoscopic mechanical oscillator and an optical pulse. We find two types of paradox, defined by whether it is the oscillator or the pulse that shows the effect Schrodinger called "steering". Only the oscillator paradox addresses the question of mesoscopic local reality for a massive system. In that case, EPR's "elements of reality" are defined for the oscillator, and it is these elements of reality that are falsified (if quantum mechanics is complete). For this sort of paradox, we show that a thermal barrier exists, meaning that a threshold level of pulse-oscillator interaction is required for a given thermal occupation $n_{0}$ of the oscillator. We find there is no equivalent thermal barrier for the entanglement of the pulse with the oscillator, nor for the EPR paradox that addresses the local reality of the optical system. Finally, we examine the possibility of an EPR paradox between two entangled oscillators. Our work highlights the asymmetrical effect of thermal noise on quantum nonlocality.
\end{abstract}

\section{INTRODUCTION}

It is an outstanding challenge in fundamental physics to test quantum nonlocality for mesoscopic, massive systems. The Einstein-Podolsky-Rosen (EPR) paradox [1] is one of the most powerful tests of quantum nonlocality. Presented originally as an argument for the completion of quantum mechanics (QM), the EPR paradox has been experimentally realised so far only in optics [2 7 ].

The observation of an EPR paradox for the position and momentum of mesoscopic mechanical oscillators would represent an important advance, since this would demonstrate the inconsistency of QM with the local reality (LR) of a massive object. First proposed by Giovannetti et al [8], such a realisation would also give an experimental platform to probe the macroscopic reality of an object, along the lines suggested by Schrodinger $[9$ 11]. While mesoscopic superpositions were achieved with ion-traps and microwave oscillators, the use of nanomechanical oscillators creates mass distribution superpositions, which tests the effects of gravity.

In light of the exceptional importance of these developments, we examine in this paper the limitations imposed by thermal noise for achieving an EPR paradox in an optomechanical system. There have been numerous proposals and studies, but mainly for the entanglement of optical and/ or mechanical modes [12 22]. Relatively little is known about the paradox itself. We expect that an EPR paradox for the positions and momentum of the mechanical oscillator will be strongly masked by thermal motion. Advances in cooling to the ground state of mesoscopic oscillators improve the likelihood of the realisation of a massive particle EPR paradox [23].

First, let us recall the important features of the EPR paradox. The original EPR state was an entangled state of two particles (which we label $A$ and $B$ ) that have perfectly correlated positions $\left(X_{A}, X_{B}\right)$ and momenta $\left(P_{A}\right.$, $\left.P_{B}\right)$. Measurements on particle A give immediate information about either the position or momentum of particle B. The EPR paradox arises because the assumption that the measurements do not disturb particle B (Local Realism) would imply a simultaneous and very precise predetermination for both of $X_{B}$ and $P_{B}$. No local quantum state of the particle $B$ however can be consistent with such precise predetermination, for both momentum and position. In this way, an inconsistency between Local Realism (LR) and the completeness of quantum mechanics is established.

The distinctive feature of the EPR paradox is that, unlike entanglement, it is a form of quantum nonlocality in which the roles of the two systems are asymmetrical. In the above example, an inconsistency of QM with LR is established for the local system B. The details about the system A - which acts only to give information about $\mathrm{B}$ - are not so important.

The main point of this paper is to understand how to obtain an irrefutable discrepancy between quantum mechanics and the local reality of the mechanical oscillator system. We propose to do this by entangling it with an optical pulse. We consider an idealised model, developed by Hofer et al 24], for pulsed optomechanics on fast time scales [25, 26]. The model introduces only two parameters: the squeeze parameter $r$ which is a measure of the pulse-oscillator interaction, and $n_{0}$, the thermal occupation number of the mechanical oscillator. Our main conclusion is that thermal noise provides a strong, but not insuperable, barrier to the oscillator EPR paradox. The barrier however is directional, to prevent "steering" of the thermally-excited mechanical system, in a sense we will explain below.

To detect the EPR paradox one must consider nonideal states, and it is not enough to simply prove entanglement. Suppose we use scaled quadratures, so that we can write the Heisenberg uncertainty relation for particle $B$ as $\Delta X_{B} \Delta P_{B} \geq 1$. Then the simplest form of an EPR paradox is realised when an inferred uncertainty relation is "violated" under the assumptions of LR, so that [27]

$$
E_{B \mid A} \equiv \Delta_{i n f} X_{B} \Delta_{i n f} P_{B}<1
$$

Here $\Delta_{i n f} X_{B}$ and $\Delta_{i n f} P_{B}$ are the uncertainties associated with the collapsed wave functions created by local 
measurements (made by "Alice") on particle $A$. These uncertainties allow her to infer either the position or momentum of particle $B$ to a given accuracy, depending on the choice of her measurements. The realisation of this EPR criterion (11) poses a more difficult challenge than the realisation of entanglement.

There has been a resurgence of interest in this area with new experiments 28 34 motivated by a realisation [35, 36] that the paradox is also an example of the nonlocality referred to as "steering" [10]. Steering gives a way to quantify how measurements by Alice can collapse the wavepacket of $B$. For a paradox achieved by condition (1), we can conclude that Alice can steer system $B$ [35, 37]. The EPR paradox therefore is a stronger test of QM than entanglement.

Our conclusion is that thermal noise $n_{0}$ provides a stronger barrier to the EPR paradox than to entanglement. We identify two sorts of EPR paradox: $E_{m \mid c}<1$ and $E_{c \mid m}<1$, where $m$ and $c$ are the oscillator and cavity field respectively. The most important is $E_{m \mid c}<1$. By analysing the "elements of reality" associated with EPR's argument, we see it is this paradox which enables a test of the mesoscopic nonlocality for the massive system.

Specifically, we find that the thermal noise $n_{0}$ of the oscillator induces a threshold for the pulse-oscillator interaction (as measured by $r$ ) if one is to observe the paradox $E_{m \mid c}<1$. In the limit of large $n_{0}$, we require $r>\frac{1}{2} \ln 2$. Consistent with the fact that the field is not thermally excited, we find there is no similar thermal barrier for an EPR paradox $E_{c \mid m}<1$, which demonstrates a "steering" of the optical system. The oscillator-pulse system therefore exhibits regimes of "one-way" steering [32, 38], where only the steering of the pulse is detectable.

We also find there is no (similar) thermal barrier for the entanglement between the optical pulse and oscillator. In this dissipation-free model, entanglement can exist for any $n_{0}$ and $r>0$. We will see however that the thermally insensitive entanglement must manifest in an asymmetric way, by measurement of the variances of quantities, $X_{A}-$ $g_{x} X_{B}, P_{A}+g_{p} P_{B}$, where $g_{x}, g_{p}$ are selected real numbers, not equal to 1 .

As a final result of the paper, we examine the possibility of an EPR paradox between two mechanical oscillators that are thermally excited. This leads us to distinguish a subclass of "symmetric" entanglement, which can be detected with $g_{x}=g_{p}=1$, and for which a thermal barrier $r>\frac{1}{2} \ln n_{0}$ does exist. We are able to show that this symmetric form of entanglement is relevant to the creation of entanglement between two symmetric thermal oscillators, and therefore has its own fundamental significance. The symmetric entanglement is detected by the criterion of Duan et al [39]. By examining two asymmetrically excited oscillators, we conclude that the "EPR steering" of one by the other can be made largely insensitive to the level of thermal excitation of one of the oscillators.

\section{THE HAWH MODEL}

We consider a mechanical oscillator coupled to an optical mode of a cavity [8]. Hofer, Wieczorek, Aspelmeyer and Hammerer (HWAH) 24] proposed a scheme (Figure 1) in which a light pulse is input to an optomechanical cavity mode and interacts with the oscillator mirror mode via radiation pressure [40]. The pulse emerges from the cavity with quadratures that are EPR correlated with those of the oscillator. The effective interaction Hamiltonian [40-42] for the cavity-oscillator system in a frame rotating at the laser frequency is

$$
H=\omega_{m} a_{m}^{\dagger} a_{m}+\Delta_{c} a_{c}^{\dagger} a_{c}+g_{R}\left(a_{m}+a_{m}^{\dagger}\right)\left(a_{c}+a_{c}^{\dagger}\right),
$$

where $\Delta_{c}=\omega_{c}-\omega_{1}$ is the detuning of the cavity with respect to the laser 24]. The boson creation and destruction operators for the optical cavity and mechanical modes are $a_{c}, a_{c}^{\dagger}$ and $a_{m}, a_{m}^{\dagger}$ respectively. Quadrature phase amplitudes $X_{c / m}, P_{c / m}$ are defined according to $a_{c}=\left(X_{c}+i P_{c}\right) / 2$ and $a_{m}=\left(X_{m}+i P_{m}\right) / 2$, where the choice of scaling ensures the normalised EPR inequality $\Delta_{\text {inf }} X_{B} \Delta_{\text {inf }} P_{B}<1$. The term in $g_{R}$ describes the linearised optomechanical coupling due to the radiation pressure, and comprises both a beam splitter-type coupling (involving $\left.a_{m}^{\dagger} a_{c}+a_{c}^{\dagger} a_{m}\right)$ and a two-mode squeezing interaction term (involving $a_{m} a_{c}+a_{m}^{\dagger} a_{c}^{\dagger}$ ) of the type

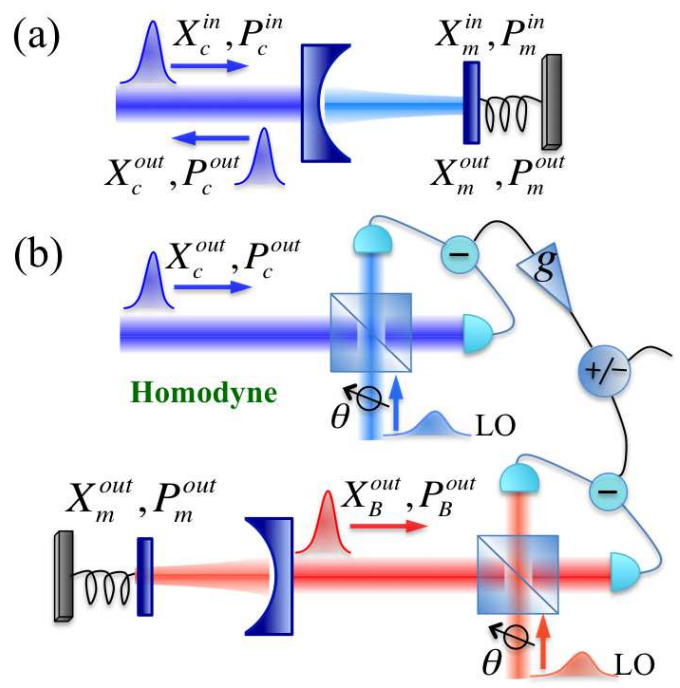

Figure 1. (Color online) Measurement of the EPR paradox between an oscillator and a pulse. (a) Entangling a pulse with a mechanical oscillator. Following HWAH, an "entangling" blue-detuned pulse interacts with an optomechanical system. The output pulse amplitudes $X_{c}^{\text {out }}, P_{c}^{\text {out }}$ are EPR correlated with the final quadratures $X_{m}^{\text {out }}, P_{m}^{\text {out }}$ of the mechanical oscillator, according to $X_{c}^{\text {out }} \sim-P_{m}^{\text {out }}$ and $P_{c}^{\text {out }} \sim-X_{m}^{\text {out }}$, in the limit of a large squeezing parameter $r$. (b) To verify the EPR paradox. The output pulse amplitudes $X_{c}^{\text {out }}, P_{c}^{\text {out }}$ are measured by homodyne detection. The quadratures of the oscillator can be measured by interacting the cavity with a second "verifying" red-detuned pulse. 
known to generate EPR entanglement [27, 43].

The physical parameters of the HAWH pulse-oscillator model are the interaction strength $g_{R}$, the oscillation frequency $\omega_{m}$, with dissipation rate $\gamma$, the optical cavity resonance frequency $\omega_{c}$ with decay rate $\kappa$, the pulse carrier frequency $\omega_{1}$ and duration time $\tau$. The initial occupation number $n_{0}$ of the thermal state of the mirror is a vital number which determines the 'quantumness' of the system. HWAH propose the pulse to be either bluedetuned or red-detuned to the cavity resonance [24], to enhance either the two-mode squeezing term (for the purpose of generating entanglement) or the beam splittertype term (for the purpose of measurement).

To generate the correlations of the EPR paradox, a blue-detuned pulse interacts with the oscillator cavity. In the case where $g_{R} \ll \kappa \ll \omega_{m}$, HWAH derive a set of idealised Langevin equations for the mode operators. To justify neglecting decoherence, they assume the pulse duration and it's interaction time with the cavity are short compared to the mechanical decoherence time. The effect of the coupling of the oscillator to an environmental heat bath is ignored.

For the blue-detuned pulse, after making a rotating wave approximation (RWA), with an adiabatic solution for the cavity mode, the simplified Langevin equations lead to solutions for quadratures $X_{c}^{\text {out }}, P_{c}^{\text {out }}$. The solutions are 24, 44]

$$
\begin{aligned}
& X_{c}^{\text {out }}=-e^{r} X_{c}^{i n}-\sqrt{e^{2 r}-1} P_{m}^{i n}, \\
& P_{c}^{\text {out }}=-e^{r} P_{c}^{i n}-\sqrt{e^{2 r}-1} X_{m}^{i n}, \\
& X_{m}^{\text {out }}=e^{r} X_{m}^{i n}+\sqrt{e^{2 r}-1} P_{c}^{i n}, \\
& P_{m}^{\text {out }}=e^{r} P_{m}^{i n}+\sqrt{e^{2 r}-1} X_{c}^{i n},
\end{aligned}
$$

where $X_{m}^{\text {out }}$ and $P_{m}^{\text {out }}$ are the final quadratures of the mechanical oscillator, and $r=g_{R}^{2} \tau / \kappa$ is the "squeezing parameter". The initial quadratures of the oscillator incorporate the effect of the thermal excitation parameter $n_{0}$.

The input-output solutions (3) are similar to those of a two-mode squeezed state [45] and will form the basis for modeling the fundamental constraints provided by the thermal noise for an EPR paradox. The solutions (3) in the limit of large $r$ become $X_{c}^{\text {out }}=-e^{r}\left(X_{c}^{i n}+P_{m}^{i n}\right)$ and $P_{c}^{\text {out }}=-e^{r}\left(P_{c}^{i n}+X_{m}^{i n}\right)$. The EPR nature of the correlations is evident, since

$$
X_{m}^{\text {out }}=-P_{c}^{\text {out }}, P_{m}^{\text {out }}=-X_{c}^{\text {out }}
$$

so that a measurement of the quadrature $X_{c}^{\text {out }}$ (or $P_{c}^{\text {out }}$ ) of the pulse will immediately give the prediction for the quadrature $-P_{m}^{\text {out }}$ (or $-X_{m}^{\text {out }}$ ) of the oscillator.

The HAWH model is very idealised, and further work is needed to test the validity of the approximations for the pulse and to model the significant decoherence expected for an oscillator interacting with its environment. The model does however capture the main physical effects that generate an EPR correlation, and gives a treatment of the thermal noise of the initial state of the oscillator.
It can be therefore be used to give a first order understanding of the asymmetrical effects of thermal noise on the EPR correlation, and of the level of thermal cooling that may be necessary, in order to observe an EPR paradox.

\section{DETECTING THE ENTANGLEMENT}

Often, entanglement is measured as a reduction in two variances, $\left\{\Delta\left(X_{m}^{\text {out }}+P_{c}^{\text {out }}\right)\right\}^{2}$ and $\left\{\Delta\left(P_{m}^{\text {out }}+X_{c}^{\text {out }}\right)\right\}^{2}$ that involve symmetric weightings of oscillator and field quadratures [39]. The symmetric criterion of Duan, Giedke, Cirac and Zoller (DGCZ) [39] detects entanglement when

$$
\left\{\Delta\left(X_{m}^{\text {out }}+P_{c}^{\text {out }}\right)\right\}^{2}+\left\{\Delta\left(P_{m}^{\text {out }}+X_{c}^{\text {out }}\right)\right\}^{2}<4
$$

where we denote the variance using the notation $\{\Delta x\}^{2} \equiv$ $\left\langle x^{2}\right\rangle-\langle x\rangle^{2}$. This criterion, however, is far from being an optimal signature for entanglement, owing to intrinsic asymmetries.

Here, we examine a less restrictive condition. Entanglement between the oscillator and pulse is proved if one can show [46, 47]

$$
\Delta_{g, \text { ent }}=\frac{\left\{\Delta\left(X_{m}^{\text {out }}+g_{x} P_{c}^{\text {out }}\right)\right\}^{2}\left\{\Delta\left(P_{m}^{\text {out }}+g_{p} X_{c}^{\text {out }}\right)\right\}^{2}}{\left[\left|g_{x} g_{p}\right|+1\right]^{2}}<1 \text {, }
$$

where $g_{x}$ and $g_{p}$ are arbitrary real numbers. The variances in the numerator are directly measurable by the scheme depicted in Fig. 1] where the $g_{x}$ and $g_{p}$ are classical gain factors. Here $\Delta_{g, e n t}$ can be minimized to a value $\Delta_{e n t}$ by choosing the optimal factor $g_{x}=g_{p}=g$

$$
g=\frac{\delta+\sqrt{\delta^{2}+4 e^{2 r}\left(e^{2 r}-1\right)}}{2 e^{r} \sqrt{e^{2 r}-1}},
$$

where $\delta=\frac{\Delta^{2} X_{m}^{i n}-\Delta^{2} P_{c}^{i n}}{\Delta^{2} X_{m}^{i n}+\Delta^{2} P_{c}^{i n}}$. We assume the initial state of the light field to be the vacuum state and that of the mirror to be a thermal state with mean excitation number $n_{0}$. In this case, $\delta=\frac{n_{0}}{n_{0}+1}$.

It is shown elsewhere [39, 48] that the condition given by (6) with optimal $g$ is equivalent to the positive partial transpose (PPT) condition developed by Simon [46] for Gaussian states, and is therefore necessary and sufficient for Gaussian two-mode entanglement. The entanglement $\Delta_{\text {ent }}$ can be measured by the arrangement of Figure 1

We note that, unlike "steering", entanglement is defined as a property that the two systems share without specification of direction - that is, if $A$ is entangled with $B$, we know that $B$ is entangled with $A$. Consistent with this, we see that the criterion (6) is symmetric with respect to interchange of $m$ and $c$, provided $g_{x}$ and $g_{p}$ are interchanged with their reciprocals. Thus, entanglement can be detected using either criterion (6), or the criterion obtained in interchanging $m$ with $c$, provided the choice of the $g_{x}$ 's and $g_{p}$ 's is kept fully flexible. The prediction 


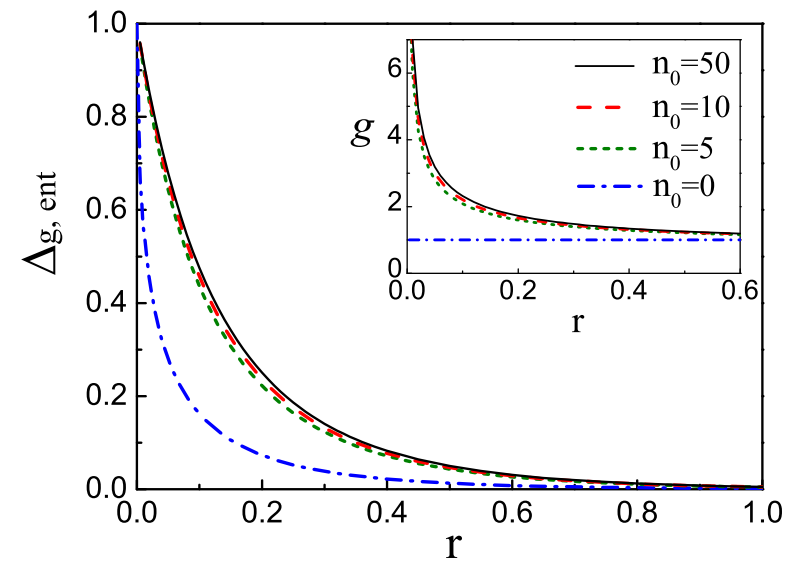

Figure 2. (Color online) Entanglement $\Delta_{g, \text { ent }}$ plotted versus the squeezing parameter $r$ for $n_{0}=0,5,10$, 50, where $n_{0}$ is initial occupation number of the oscillator. Entanglement between the oscillator and pulse is observed when $\Delta_{\text {ent }}<1$. Strong entanglement occurs when $\Delta_{\text {ent }} \rightarrow 0$. The optimal $g$ to minimize $\Delta_{g \text {,ent }}$ is shown in the inset. The results indicate presence of entanglement, even for large $n_{0}$.

of the model (3) for the entanglement measured by $\Delta_{g, \text { ent }}$ is plotted in Fig. 2

Now we come to the first important result of this paper. Surprisingly, we see from the Fig. 2 that no thermal barrier exists for the presence of entanglement. For any given thermal occupation number $n_{0}$, we can always show entanglement for $r>0$. In other words, once the system is entangled, no amount of thermal noise can completely destroy it. A similar type of robustness of entanglement has been predicted for the steady state opto-mechanical entanglement that is generated using continuous wave light fields [12 15]. We note from the Figure that the detection of this thermally insensitive entanglement is linked to values of the parameter $g_{\text {opt }}$ that are very different to 1. As we will see below, this result can be understood in terms of the concept of quantum steering.

This result contrasts with that obtained for entanglement detected using the symmetric DGCZ condition (5) [39], given by $\Delta_{g, \text { ent }}<1$ where $g_{x}=g_{p}=1$. We call this sort of entanglement "symmetric entanglement". In that case, a thermal barrier does exist, and entanglement can be detected only when the squeezing parameter is sufficiently large [24],

$$
r>\ln \frac{n_{0}+2}{2 \sqrt{n_{0}+1}} \stackrel{n_{0} \rightarrow \infty}{\sim} \frac{1}{2} \ln n_{0} .
$$

While this thermal barrier becomes relevant to detecting entanglement between two symmetric, thermally excited oscillators, it does not place a limit on the detection of entanglement between the oscillator and a pulse.

\section{DETECTING AN EPR PARADOX AND QUANTUM STEERING}

\section{A. Quantum steering of the mechanical oscillator by the pulse}

Now we examine how to detect an EPR paradox. An EPR paradox is confirmed if [27]

$$
E_{m \mid c}=\Delta_{i n f} X_{m}^{o u t} \Delta_{i n f} P_{m}^{o u t}<1,
$$

where $\Delta_{\text {inf }} X_{m}^{\text {out }}$ is the error in the prediction for the value of the oscillator's position, made by a measurement on the pulse. The $\Delta_{\text {inf }} P_{m}^{\text {out }}$ is defined similarly. The realisation of $E_{m \mid c}<1$ is verification of a quantum steering of the mechanical system by measurements made on the pulse [35, 37]. For the subclass of quantum systems given by Gaussian states and measurements, as is the case here, this criterion becomes necessary and sufficient to detect steering of the system $m$ by the second system [35]. Walborn et al have derived a more sensitive entropic criterion for "EPR steering" that is useful in other cases [49].

A simple way to determine the conditional uncertainties for Gaussian distributions is to use a linear estimate $g_{x} P_{c}^{\text {out }}$, based on the result $P_{c}^{\text {out }}$ for measurement at $A$ [5, 27]. We find

$$
\Delta_{\text {inf }} X_{m}^{\text {out }}=\Delta\left(X_{m}^{\text {out }}-g_{x} P_{c}^{\text {out }}\right),
$$

where $g_{x}=\left\langle X_{m}^{\text {out }}, P_{c}^{\text {out }}\right\rangle /\left\{\Delta P_{c}^{\text {out }}\right\}^{2}$ is optimised to minimise $\left\{\Delta_{\text {inf }} X_{m}^{\text {out }}\right\}^{2}$. Here we use the notation $\langle x, y\rangle \equiv\langle x y\rangle-\langle x\rangle\langle y\rangle$. Similarly, the conditional variance $\left\{\Delta_{\text {inf }} P_{m}^{\text {out }}\right\}^{2}$ is evaluated by

$$
\left\{\Delta_{\text {inf }} P_{m}^{\text {out }}\right\}^{2}=\left\{\Delta\left(P_{m}^{\text {out }}+g_{p} X_{c}^{\text {out }}\right)\right\}^{2},
$$

where $g_{p}=\left\langle P_{m}^{\text {out }}, X_{c}^{\text {out }}\right\rangle /\left\{\Delta X_{c}^{\text {out }}\right\}^{2}$. We note that the values of $g_{x}, g_{p}$ that optimise for the EPR paradox are generally different to those that optimise the entanglement given by (6).

We assume the light to be initially in a vacuum state and the mechanical oscillator to be initially in a thermal state, with mean occupation number $n_{0}$. We can then calculate the prediction of the model (3) for the EPR paradox. We find

$$
\Delta_{i n f} X_{m}^{\text {out }}=\Delta_{\text {inf }} P_{m}^{\text {out }},
$$

where we take $g_{x}=g_{p}=g$ and

$$
g=\frac{2 e^{r} \sqrt{e^{2 r}-1}\left(n_{0}+1\right)}{2 e^{2 r}\left(n_{0}+1\right)-\left(2 n_{0}+1\right)} .
$$

The EPR paradox parameter $E_{m \mid c}$ is given in the Fig. 3 versus $r$, for various values of initial thermal occupation $n_{0}$. The EPR paradox realised when $E_{m \mid c}<1$ is predicted for values of squeezing parameter given by

$$
r>r_{\text {epr }}=\frac{1}{2} \ln \frac{2 n_{0}+1}{n_{0}+1} \stackrel{n_{0} \rightarrow \infty}{\sim} \frac{1}{2} \ln 2 .
$$


There is a temperature-dependent minimal squeezing parameter $r_{e p r}$ required to observe the paradox. We note however that for large thermal excitation $n_{0}$, the barrier becomes fixed, at $r_{e p r}=\frac{1}{2} \ln 2$ as $n_{0} \rightarrow \infty$.

This is the second noteworthy result. If the squeeze parameter $r$ is large enough (that is, if there is enough entanglement between the oscillator and the pulse), the quantum steering of the oscillator by the pulse cannot be destroyed by further increasing the thermal noise value $n_{0}$. The quantum steering of the oscillator by the pulse can be achieved when $r>\frac{1}{2} \ln 2$. We expect that this effect arises because the second EPR system, the pulse, is not thermally excited. A different effect is expected for the EPR paradox between two oscillators.

\section{B. Quantum steering of the pulse by the oscillator}

An EPR paradox can be shown the other way, by the criterion

$$
E_{c \mid m}=\Delta_{i n f} X_{c}^{\text {out }} \Delta_{\text {inf }} P_{c}^{\text {out }}<1
$$

In this case, the optical pulse is "steered" by the measurements made on the mechanical oscillator. Results for the prediction of $E_{c \mid m}$ based on the model (3) are shown in Fig. 3 Such an EPR paradox is thermally insensitive, being possible for any value of initial thermal noise $n_{0}$, and for any squeezing parameter $r>0$. Since EPR steering requires entanglement [35], this property underpins the thermal insensitivity noted above for entanglement. However, because the thermally insensitive steering is "one-way" only, it does not correspond to an entanglement that can be detected symmetrically with respect to the oscillators - that is, with $g=1$.

We note that there are two regimes for the observation of quantum steering and the EPR paradox. For $r \leq \frac{1}{2} \ln 2$, the only EPR paradox possible is $E_{c \mid m}<1$ ("one-way steering" $30-32,50]$ ). For $r>\frac{1}{2} \ln 2$, "two-way steering" becomes possible, and both paradoxes $E_{m \mid c}<1$ or $E_{c \mid m}<1$ can be confirmed.

Now we can understand the reason for the reduced sensitivity of the entanglement to the thermal noise of the oscillator. We have shown that a threshold squeezing parameter $r$ is necessary to enable a steering of the thermal oscillator by the measurements made on the pulse, but there is no threshold for the steering of the pulse by the measurements on the oscillator. Entanglement is a defined as a shared quantity, and must exist between the two systems if either form of steering is achieved [35]. Hence, entanglement is detected without the threshold, because this is possible for the quantum steering $\left(E_{c \mid m}<1\right)$ in one direction.

We argue however that the symmetric form of entanglement has its own special significance. The DGCZ criterion (5) is defined as that entanglement detected in a symmetric way, with $g_{x}=g_{p}=1$. This distinguishes it from the thermally insensitive entanglement that is detected with $g_{x}, g_{p}$ values very different to 1 (Fig. 3).

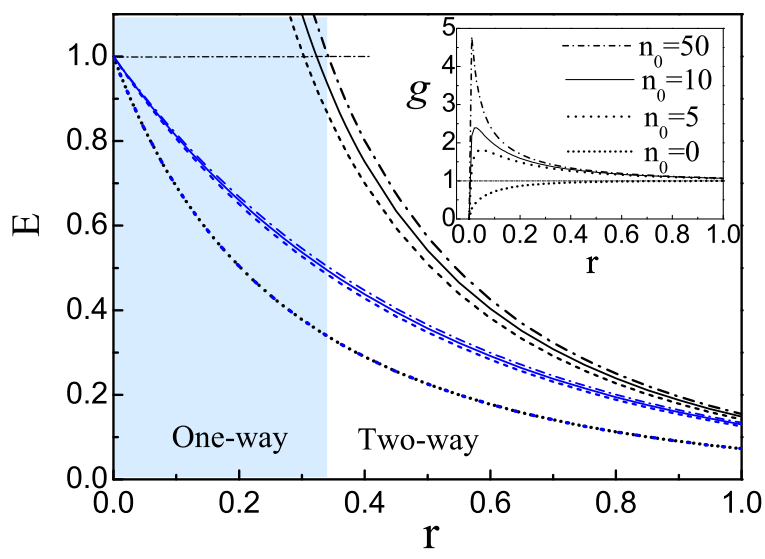

Figure 3. (Color online) EPR paradox and quantum steering between the oscillator and the pulse. An EPR paradox and quantum steering of the oscillator by the pulse is detected when $E=E_{m \mid c}<1$ (black upper set of lines, that intercept with the line $E=1$ at the value for $r$ given by $r_{e p r}$ of Eq. (14)). An EPR paradox and the steering of the pulse by the oscillator is detected when $E=E_{c \mid m}<1$ (blue lower set of lines). The lowest two superposed curves show $E_{m \mid c}$ and $E_{c \mid m}$ with $n_{0}=0$ (dotted). The inset shows the optimal $g$ to minimize $E_{m \mid c}$.

For symmetric systems, like two equivalently thermally excited oscillators, we conjecture that the limitations for entanglement are determined by the DGCZ criterion.

\section{FUNDAMENTAL SIGNIFICANCE OF THE STEERING OF THE OSCILLATOR}

To understand the importance of the EPR paradox that demonstrates quantum steering of the oscillator by the pulse, we first review the meaning of an EPR paradox [1]. An EPR paradox arises because the assumption of local realism (LR) would imply a simultaneous, precise predetermination for both of $X_{m}^{\text {out }}$ and $P_{m}^{\text {out }}$. No quantum state of the oscillator however can be consistent with such a predetermination, for both momentum and position. Thus, EPR argue "elements of reality" exist that cannot be described by quantum mechanics, and an inconsistency between LR and the completeness of quantum mechanics is revealed.

We have found there is a thermal barrier for the EPR paradox that corresponds to the "steering" of the oscillator system. This will make this sort of paradox more difficult to observe in practice. We note however that there is a fundamental significance in observing this sort of EPR paradox. On examining the EPR argument, we see that the EPR paradox (in this case) is based on the premise that the action of measuring the pulse cannot change the state of the oscillator. Hence, "elements of reality" are deduced for the oscillator system (not the pulse). These "elements of reality" become inconsistent with quantum mechanics when $E_{m \mid c}=\Delta_{i n f} X_{m} \Delta_{i n f} P_{m}<1$. Hence, 
(a)

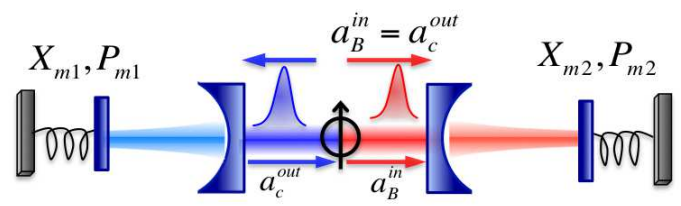

(b)

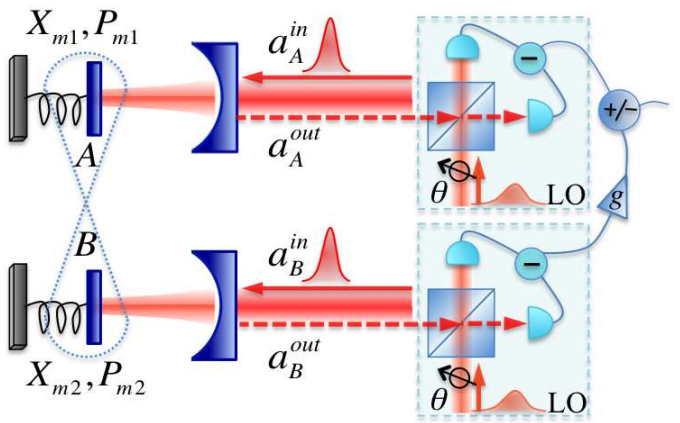

Figure 4. (Color online) Entangling two oscillators: (a) Generation of the entanglement takes place when the output of the first cavity is injected into the second cavity, as red-detuned. The final states of the two oscillators at $A$ and $B$ will be entangled. (b) The entanglement can be verified, at a later stage, using two red detuned pulses, and the homodyne scheme set up as depicted, to measure the conditional inference variances $\left\{\Delta_{i n f} X_{m j}\right\}^{2}$ and $\left\{\Delta_{i n f} P_{m j}\right\}^{2}$.

if local realism LR is correct, the oscillator cannot be described quantum mechanically. Alternatively, with an assumption that quantum mechanics is complete, it is the local reality of the oscillator that is disproved. For the second type of paradox, it is the reality of the optical state that is addressed. This is less useful for direct insights about quantum effects with matter.

\section{ENTANGLEMENT AND EPR PARADOX BETWEEN TWO MECHANICAL OSCILLATORS}

A further challenge is to understand the thermal limits for obtaining an EPR paradox between two thermally excited mechanical oscillators. Bipartite entanglement between two mechanical oscillators can be achieved, in principle, by "swapping" the entanglement between the oscillator $m 1$ and the output pulse, to an entanglement between the oscillator $m 1$ at $A$ and a second mechanical oscillator $m 2$ at location $B$. Other methods are possible, the simplest being to couple two cavities to two incoming entangled light fields [16-22]. Most previous calculations have been limited to the generation of entanglement in steady state regimes.

In this paper, we focus for simplicity on the results of calculations based on the first method (Fig. 4). After interaction with the first cavity, the entangling pulse "carries" the information about the quadratures of the first oscillator. As $r \rightarrow \infty$, we see from (3) that $X_{m 1}^{\text {out }}=-P_{c}^{\text {out }}$ and $P_{m 1}^{o u t}=-X_{c}^{\text {out }}$. Suppose then that after the coupling to the first cavity and oscillator $m 1$, the output entangling pulse is then red-detuned relative to a second mechanical oscillator $(m 2)$ and cavity system. After an interaction with this pulse, the final amplitudes of the second oscillator are [24]

$$
\begin{aligned}
& X_{m 2}^{\text {out }}=e^{-r^{\prime}} X_{m 2}^{\text {in }}+\sqrt{1-e^{-2 r^{\prime}}} P_{c}^{\text {out }}, \\
& P_{m 2}^{\text {out }}=e^{-r^{\prime}} P_{m 2}^{\text {in }}-\sqrt{1-e^{-2 r^{\prime}}} X_{c}^{\text {out }},
\end{aligned}
$$

where $r^{\prime}$ is the squeezing parameter of the second cavity, and $P_{c}^{\text {out }}, X_{c}^{\text {out }}$ are given by (3). As $r^{\prime} \rightarrow \infty$, the relations between the quadratures of the second mechanical oscillator and the pulse are $X_{m 2}^{\text {out }}=P_{c}^{o u t}$ and $P_{m 2}^{\text {out }}=-X_{c}^{\text {out }}$, which will "swap" the EPR correlation $\Delta\left(P_{c}^{\text {out }}+g X_{m 1}^{\text {out }}\right), \Delta\left(X_{m 1}^{\text {out }}-g P_{c}^{\text {out }}\right)$ into an EPR correlation $\Delta\left(X_{m 2}^{\text {out }}+g X_{m 1}^{\text {out }}\right), \Delta\left(X_{m 1}^{\text {out }}-g X_{m 2}^{\text {out }}\right)$ between the mechanical oscillators. Thus, an EPR paradox between between the pulse and the first mechanical oscillator is directly transformed into an EPR paradox between two mechanical oscillators in the limit of $r^{\prime} \gg r$ and $r^{\prime} \rightarrow \infty$.

For practical reasons, since the thermal noise on the second oscillator can be significant, it is also informative to consider definite predictions for $r=r^{\prime}$. The final entanglement and EPR paradox variances are readily calculated. Solving, we find

$$
\begin{aligned}
\left\{\Delta_{\text {inf }} X_{m 2}^{\text {out }}\right\}^{2}= & \left\{\Delta\left(X_{m 2}^{\text {out }}+g X_{m 1}^{\text {out }}\right)\right\}^{2} \\
= & e^{-2 r}\left\{\Delta X_{m 2}^{\text {in }}\right\}^{2}+(g-1)^{2}\left(e^{2 r}-1\right)\left\{\Delta P_{c}^{\text {in }}\right\}^{2} \\
& \quad+\left[(g-1) e^{r}+e^{-r}\right]^{2}\left\{\Delta X_{m 1}^{\text {in }}\right\}^{2} \\
= & e^{-2 r}\left(2 n_{m 2}+1\right)+(g-1)^{2}\left(e^{2 r}-1\right) \\
& \quad+\left[(g-1) e^{r}+e^{-r}\right]^{2}\left(2 n_{m 1}+1\right)
\end{aligned}
$$

and $\Delta_{\text {inf }} P_{m 2}^{\text {out }}=\Delta\left(P_{m 2}^{\text {out }}-g P_{m 1}^{\text {out }}\right)=\Delta_{\text {inf }} X_{m 2}^{\text {out }}$. Here, $n_{m 1}, n_{m 2}$ are the thermal occupation numbers for the two oscillators.

\section{A. Entanglement}

Importantly, we note that a thermal barrier exists for the entanglement between two oscillators with equal thermal noise $n_{m 1}=n_{m 2}=n_{0}$. We examine the predictions for the entanglement criterion (6) but as applied to the two oscillators $m 1$ and $m 2$. In this case, the optimal $g$ for the detection of entanglement (given by $\frac{\partial \Delta_{g, e n t}}{\partial g}=0$ ) is

$$
\begin{aligned}
g= & \sqrt{1+\left(2 n_{0}+1\right)^{2} / 4 e^{4 r}\left(n_{0}+1\right)^{2}} \\
& -\left(2 n_{0}+1\right) / 2 e^{2 r}\left(n_{0}+1\right)
\end{aligned}
$$

which becomes $g \rightarrow 1$ in the limit of large $n_{0}$ and $r$. The threshold squeezing parameter for entanglement becomes $r_{\text {ent }}=\frac{1}{2} \ln 2 n_{0}$ in this limit, which has the same large $n_{0}$ dependence as for the symmetric entanglement that is detected by the DGCZ entanglement criterion. The similarity is expected for two equivalent oscillators, since any entanglement that can be created between the two oscillators must be symmetric i.e. unchanged on interchange $A \longleftrightarrow B$, which will require $g_{o p t}=g_{x}=g_{p}=1$. 


\section{B. EPR paradox and steering}

We now consider the EPR paradox and quantum steering, in particular how the oscillator $m 1$ "steers" the oscillator $m 2$. The optimal $g$ for the detection of the EPR paradox $E_{m 2 \mid m 1}$ is given by $\frac{\partial \Delta_{i n f} X_{m 2}^{\text {out }}}{\partial g}=0$. Solving gives

$$
g=\frac{\left(e^{2 r}-1\right)\left(n_{m 1}+1\right)}{e^{2 r}\left(n_{m 1}+1\right)-1 / 2} .
$$

The threshold squeezing parameter for the EPR paradox $E_{m 2 \mid m 1}$ is then

$r_{e p r}=\frac{1}{2} \ln \left(n_{m 2}+1+\sqrt{\left(n_{m 2}+1\right)^{2}-\frac{n_{m 1}+n_{m 2}+1}{n_{m 1}+1}}\right)$.

The threshold $r_{e p r}$ is plotted in Fig. 53.

We find that the "steering" of oscillator $m 2$ by measurements on oscillator $m 1$ is very sensitive to the noise $n_{m 2}$ on $m 2$, and depends logarithmically on $n_{m 2}$ in the limit $n_{m 2} \rightarrow \infty$, but is insensitive to the noise $n_{m 1}$ on $m 1$. For $n_{m 2}=0, r_{e p r}=0$ and no thermal barrier exists, which gives a similar behaviour to that of the hybrid paradox $E_{c \mid m 1}$. We note however that for $n_{m 1}=0$, a thermal barrier does exist and the threshold is given by $r_{e p r} \rightarrow \frac{1}{2} \ln 2 n_{m 2}$ as $n_{m 2} \rightarrow \infty$. This gives a different sort of behaviour to that of the hybrid paradox $E_{m 1 \mid c}$. In that case, the threshold for the steering of the oscillator (by a noiseless pulse) was fixed at $r_{\text {epr }}=\frac{1}{2} \ln 2$ as the thermal noise of the oscillator increased. In this way, we learn that whether a thermally insensitive threshold exists for the steering of a mechanical oscillator will depend on the nature of entanglement preparation.

We can also consider the steering of the oscillator $m 1$ by $m 2$, which is the EPR paradox obtained when $E_{m 1 \mid m 2}<1$. We find

$$
\begin{aligned}
\left\{\Delta\left(X_{m 1}^{\text {out }}+g X_{m 2}^{\text {out }}\right)\right\}^{2}= & {\left[e^{r}-g\left(e^{r}-e^{-r}\right)\right]^{2}\left\{\Delta X_{m 1}^{\text {in }}\right\}^{2} } \\
& +(1-g)^{2}\left(e^{2 r}-1\right)\left\{\Delta P_{c}^{i n}\right\}^{2} \\
& +g^{2} e^{-2 r}\left\{\Delta X_{m 2}^{i n}\right\}^{2},
\end{aligned}
$$

and $\Delta_{\text {inf }} P_{m 1}^{\text {out }}=\Delta\left(P_{m 1}^{\text {out }}-g P_{m 2}^{\text {out }}\right)=$ $\Delta\left(X_{m 1}^{\text {out }}+g X_{m 2}^{\text {out }}\right)=\Delta_{\text {inf }} X_{m 1}^{\text {out }}$. The optimal $g$ for the detection of $E_{m 1 \mid m 2}<1$ is given by $\frac{\partial \Delta_{i n f} X_{m 1}^{\text {out }}}{\partial g}=0$. Solving gives

$$
\begin{aligned}
g= & \left(e^{2 r}-1\right)\left(\left\{\Delta X_{m 1}^{i n}\right\}^{2}+\left\{\Delta P_{c}^{i n}\right\}^{2}\right) /\left\{\left(e^{2 r}+e^{-2 r}-2\right)\right. \\
& \left.\times\left\{\Delta X_{m 1}^{i n}\right\}^{2}+\left(e^{2 r}-1\right)\left\{\Delta P_{c}^{i n}\right\}^{2}+e^{-2 r}\left\{\Delta X_{m 2}^{i n}\right\}^{2}\right\}
\end{aligned}
$$

In this case, a thermally insensitive barrier to steering does exist i.e. the threshold for the steering of oscillator $m 1$ becomes insensitive to $n_{m 1}$, as $n_{m 1} \rightarrow \infty$. In fact, as
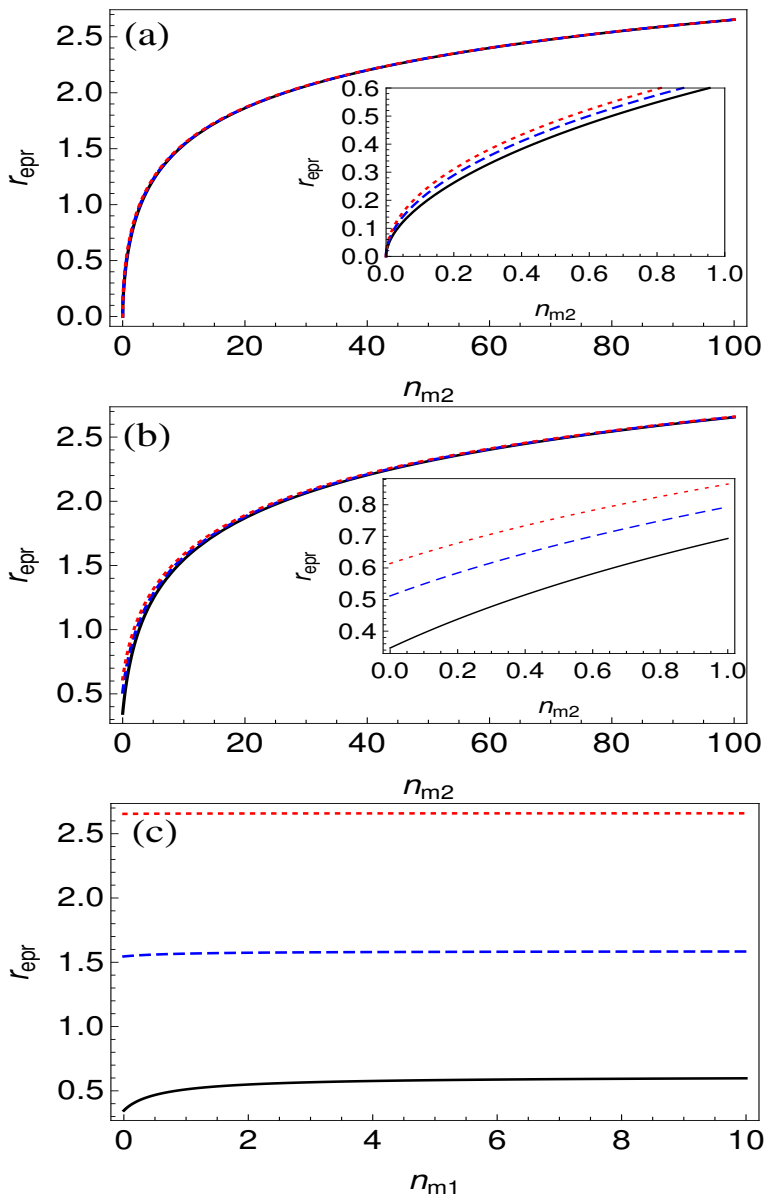

Figure 5. (Color online) Detecting an EPR paradox between two mechanical oscillators prepared using the scheme of Eqs. (16) with $r=r^{\prime}$ : (a) The threshold squeeze parameter $r_{e p r}$ for observation of the EPR paradox $E_{m 2 \mid m 1}<1$, versus the thermal occupation number of the oscillator $m 2$. The curves are for $n_{m 1}=0$ (solid), $n_{m 1}=1$ (dashed), $n_{m 1}=1.5 \times 10^{6}$ (dotted) (b) The threshold squeeze parameter $r_{\text {epr }}$ for observation of the EPR paradox $E_{m 1 \mid m 2}<1$, versus the thermal occupation number of the oscillator $m 2$. The curves are for $n_{m 1}=0$ (solid), $n_{m 1}=1$ (dashed), $n_{m 1}=1.5 \times 10^{6}$ (dotted).

(c) The threshold squeeze parameter $r_{e p r}$ for observation of the EPR paradox $E_{m 1 \mid m 2}<1$, versus the thermal occupation number of the oscillator $m 1$. The curves are for $n_{m 2}=0$ (solid), $n_{m 2}=10$ (dashed), $n_{m 2}=100($ dotted $)$

$n_{m 1} \rightarrow \infty$,

$$
\begin{aligned}
r_{e p r} & =\frac{1}{2} \ln \left(n_{m 2}+2-\frac{1}{2\left(1+n_{m 1}\right)}\right. \\
& +\sqrt{\left.\left[n_{m 2}+2-\frac{1}{2\left(1+n_{m 1}\right)}\right]^{2}-\frac{2 n_{m 2}}{1+n_{m 1}}-2\right)} \\
& \rightarrow \frac{1}{2} \ln 2 n_{m 2}
\end{aligned}
$$


as evident in the plots of Fig. [5 and c. For $n_{m 2}=0$,

$$
r_{e p r}=\frac{1}{2} \ln \left[2-\frac{1}{2\left(1+n_{m 1}\right)}+\sqrt{\left.\left[2-\frac{1}{2\left(1+n_{m 1}\right)}\right]^{2}-2\right]},\right.
$$

which approaches a fixed value as $n_{m 1} \rightarrow \infty$, consistent with the result for the threshold $r_{e p r}$ for the steering of the oscillator $m 1$ by the pulse. However, we note now (different to the result for $E_{m 2 \mid m 1}$ ) that there is a sensitivity to the noise $n_{m 2}$ of the "steering" system. The limiting value of $r_{e p r}$ increases with $n_{m 2}$. For $n_{m 1}=0$, the optimal $g$ given by Eq. (22) corresponds to a threshold squeezing parameter of $r_{e p r}=\frac{1}{2} \ln 2 n_{m 2}$ in the limit of large $n_{m 2}$.

In short, the steering between the two oscillators will become limited by the thermal noise on them. With a certain method of entanglement preparation, the steering threshold depends logarithmically on the thermal noise of the system being steered. In this case, there is very little dependence on the thermal noise of the steering system. If the entanglement is prepared another way, an oscillator can be steered (in the large thermal limit) independently of its own thermal noise, but then the steering threshold becomes sensitive logarithmically to large levels of thermal noise on the steering system.

If we consider equal thermal noise levels $n_{0}$ for the two oscillators, the thermal barrier for the quantum steering (of either oscillator) remains sensitive to the thermal noise $n_{0}$ in the limit of large $r$ : the threshold becomes $r>\frac{1}{2} \ln 4 n_{0}$. This tells us that enough thermal noise will destroy the possibility of an EPR paradox, for any given squeeze parameter $r$ that creates the entanglement.

The interesting feature noticed for this method of entanglement generation is that both the steering of oscillator $m 2$ by $m 1$, and the steering of $m 1$ by $m 2$, show an insensitivity to the thermal excitation level $n_{1}$ of oscillator $m 1$. Thus, the "steering" of an oscillator $M$ by another $S$ can be largely insensitive to the thermal excitation of $M$, or largely insensitive to the excitation of $S$, depending on the method of entanglement.

\section{CONCLUSION}

In summary, we have examined the effect of an initial thermal excitation of an oscillator on observing an EPR paradox between a mesoscopic mechanical oscillator and a pulse. A thermal barrier exists for an EPR paradox that can demonstrate a quantum "steering" of the mechanical oscillator. This is the most interesting paradox, since it tests local reality for a massive, mesoscopic system.

No equivalent thermal barrier exists for the EPR paradox that can demonstrate a "steering" of the optical pulse. Similarly, as must be the case given that all types of steering require entanglement, no barrier exists for the entanglement between the oscillator and the pulse. This robust pulse-oscillator entanglement is only detectable using fully sensitive entanglement criteria, such as obtained by the positive partial transpose PPT method.

Importantly, the thermal barrier to the steering of the oscillator by the pulse is not insurmountable: it can be overcome for a large enough squeezing parameter. For temperatures above $100 \mathrm{mK}$, the condition is $r>0.4$. This is much more favourable than the conditions $r>2.4$ at $T \sim 100 \mathrm{mK}$, and $r>7$ at $T=293 \mathrm{~K}$, required for observation of the symmetrically measured entanglement (where $g=1$ ) 24].

Our results show that the thermal noise of the mechanical object destroys the manifestation of that paradox, and that the thermal barrier increases with initial thermal excitation number $n_{0}$ if we consider the paradox between two symmetric oscillators. This gives an explanation of the difficulty of observing mesoscopic EPR paradox effects between massive oscillators at room temperature.

We show that a very big advantage is to be gained if we consider the EPR paradox of an oscillator with an optical field, which is not thermally excited, since then (for this simple model) the threshold interaction to show quantum steering of the oscillator becomes fixed for $n_{0} \gg 1$. By analysing an entanglement swapping scenario that leads to an entanglement of two thermally excited oscillators, we show that this advantage is lost for the EPR paradox between two symmetric oscillators.

We conclude by commenting that a practical prediction for the EPR paradox must fully incorporate the main sources of decoherence. The practical limitation is that larger pulse-cavity interaction times lead to increased mechanical decoherence, due to the coupling to the environmental heatbath at temperature $T_{\text {bath }}$. The results presented here are based on an idealised model which ignores the coupling to the heat bath of the environment. More complete models have been given in Ref. [24]. Those authors did not however analyse the effects of an environmental heat bath on the EPR paradox, but restricted to a study of the symmetric DGCZ entanglement. Nonetheless, they estimated that the symmetric entanglement is feasible, provided $Q f \gg k_{B} T_{b a t h} / h$, where $T_{b a t h}$ is the temperature of the environment, $f$ is the frequency of mechanical vibration and $Q$ is the cavity quality. Based on the results of this paper, more optimistic predictions would be expected, both for entanglement as detected by the PPT condition, and also for an EPR paradox.

\section{ACKNOWLEDGMENTS}

We thank P. Drummond, W. Bowen and S. Hofer for useful discussions and information. We acknowledge support from the Australian Research Council via Discovery and DECRA grants. Q. Y. H. thanks the National Natural Science Foundation of China under Grant No. 11121091 and 11274025. 
[1] A. Einstein, B. Podolsky, and N. Rosen, Phys. Rev. 47, 777 (1935).

[2] C. S. Wu and I. Shaknov, Phys. Rev. 77, 136 (1950).

[3] A. Aspect, P. Grangier, and G. Roger, Phys. Rev. Lett. 49, 91 (1982).

[4] Z. Y. Ou, S. F. Pereira, H. J. Kimble, and K. C. Peng, Phys. Rev. Lett. 68, 3663 (1992).

[5] M. D. Reid et al., Rev. Mod. Phys. 81, 1727 (2009) and experiments referenced therein.

[6] J. Howell, R. Bennink, S. Bentley, and R. Boyd, Phys. Rev. Lett. 92, 210403 (2004).

[7] B. Hage, A. Samblowski, and R. Schnabel, Phys. Rev. A 81, 062301 (2010); T. Eberle et al., Phys. Rev. A 83, 052329 (2011); S. Steinlechner et al., Phys. Rev. A 87, 022104 (2013).

[8] V. Giovannetti, S. Mancini and P. Tombesi, Europhys. Lett. 54, 559 (2001).

[9] E. Schrï̈œdinger, Naturwissenschaften 23, 844 (1935).

[10] E. Schrį̈œdinger, Proc. Cambridge Philos. Soc. 31, 553 (1935); ibid. 32, 446 (1936).

[11] W. Marshall, R. Simon, R. Penrose and D. Bouwmeester, Phys Rev Lett, 91, 130401 (2003).

[12] D. Vitali et al., Phys. Rev. Lett. 98, 030405 (2007).

[13] C. Genes, A. Mari, P. Tombesi and D. Vitali, Phys. Rev. A 78, 032316 (2008)

[14] C. Genes, A. Mari, D. Vitali and P. Tombesi, Adv. At. Mol. Opt. Phys. 57, 33 (2009).

[15] M. Abdi et al., Phys. Rev. A 84, 032325 (2011).

[16] J. Zhang, K. Peng, and S. L. Braunstein, Phys. Rev. A 68, 013808 (2003).

[17] M. Pinard et al., Europhys. Lett. 72, 747 (2005).

[18] M. Paternostro et al., Phys. Rev. Lett. 99, 250401 (2007).

[19] H. Miao, S. Danilishin, and Y. Chen, Phys. Rev. A 81, 05237 (2010)

[20] S. Pirandola, D. Vitali, P. Tombesi, and S. Lloyd, Phys. Rev. Lett. 97, 150403 (2006).

[21] M. Bhattacharya and P. Meystre, Phys. Rev. Lett. 99, 073601 (2007).

[22] D. Vitali, S. Mancini, and P. Tombesi, J. Phys. A: Math. Theor. 40, 8055 (2007)

[23] J. Teufel et al., Nature 475, 359 (2010); J Chan et al., Nature 478, 89 (2011); A. D. O' Connell et al., Nature 464, 697 (2010); J. Teufel et al., Nature 471, 204 (2011); S. Groblacher et al., Nature 478, 359 (2010); E. Verhagen et al., Nature 482, 63 (2012).

[24] S. G. Hofer, W. Wieczorek, M. Aspelmeyer and K. Ham- merer, Phys. Rev. A 84, 052327 (2011).

[25] M. R. Vanner et al., Proc. Nat. Ac. Sc. 108, 16182 (2011).

[26] I. Pikovski et al., Nat. Phys. 8, 393 (2012).

[27] M. D. Reid, Phys. Rev. A 40, 913 (1989).

[28] D. J. Saunders, S. J. Jones, H. M. Wiseman, and G. J. Pryde, Nature Physics 6, 845 (2010).

[29] D. H. Smith et al., Nature Commu. 3, 625 (2012).

[30] A. J. Bennet et al., Phys. Rev. X 2, 031003 (2012).

[31] B. Wittmann et al., New J. Phys. 14, 053030 (2012).

[32] V. Hï ¿œndchen et al., Nature Photonics 6, 598 (2012).

[33] J. Schneeloch et al., Phys. Rev. Lett. 110, 130407 (2013).

[34] J. Leach, B. Jack, J. Romero, A. Jha, A. Yao, S. FrankeArnold, D. G. Ireland, R. W. Boyd, S. M. Barnett, and M. J. Padgett, Science 329, 662 (2010).

[35] H. M. Wiseman, S. J. Jones, and A. C. Doherty, Phys. Rev. Lett. 98, 140402 (2007).

[36] S. J. Jones, H. M. Wiseman and A. C. Doherty, Phys. Rev. A 76, 052116 (2007).

[37] E. G. Cavalcanti, S. J. Jones, H. M. Wiseman and M. D. Reid, Phys. Rev. A 80, 032112 (2009).

[38] S. Midgley, A. J. Ferris and M. K. Olsen, Phys. Rev. A 81, 022101 (2010).

[39] L. M. Duan, G. Giedke, J. I. Cirac, and P. Zoller, Phys. Rev. Lett. 84, 2722 (2000).

[40] S. Mancini and P. Tombesi, Phys. Rev. A 49, 4055 (1994).

[41] C. K. Law, Phys. Rev. A 51, 2537 (1995).

[42] S. Mancini et al., Phys. Rev. Lett. 88, 120401 (2002).

[43] M. D. Reid and P. D. Drummond, Phys. Rev. Lett. 60, 2731 (1988).

[44] C. W. Gardiner and M. J. Collett, Phys. Rev. A 31, 3761 (1985).

[45] B. L. Schumaker and C. M. Caves, Phys. Rev. A 31, 3093 (1985). R. E. Slusher et al., Phys. Rev. Lett. 55, 2409 (1985). M. D. Reid and D. F. Walls, Phys. Rev. A 33, 4465 (1986).

[46] R. Simon, Phys. Rev. Lett. 84, 2726 (2000).

[47] V. Giovannetti, S. Mancini, D. Vitali, and P. Tombesi, Phys. Rev. A 67, 022320 (2003).

[48] Q. Y. He and M. D. Reid, to be published.

[49] S. P. Walborn, A. Salles, R. M. Gomes, F. Toscano, and P. H. Souto Ribeiro, Phys. Rev. Lett. 106, 130402 (2011).

[50] E. G. Cavalcanti, Q. Y. He, M. D. Reid and H. M. Wiseman, Phys. Rev. A 84, 032115 (2011). K. Wagner et al., arXiv:1203.1980 [quant-ph]. 